\title{
Duration of Adalimumab Therapy in Hidradenitis Suppurativa With and Without Oral Immunosuppressants
}

\author{
Micaella R. Zubkov, MD; Reid A. Waldman, MD; Rong Wu, PhD; Jane M. Grant-Kels, MD
}

\section{PRACTICE POINTS}

- Adalimumab is the only medication approved by the US Food and Drug Administration for treatment of hidradenitis suppurativa (HS), yet many patients on adalimumab do not achieve satisfactory results. New treatment options are in demand for patients affected by HS.

- Although combining tumor necrosis factor $\alpha$ inhibitors with oral immunosuppressants such as methotrexate and mycophenolate mofetil appears to be beneficial in treating other conditions such as psoriasis, these treatments may not have as great a benefit for patients with HS.

To the Editor:

The tumor necrosis factor $\alpha$ inhibitor adalimumab is the only US Food and Drug Administration-approved treatment of hidradenitis suppurativa (HS). Although 50.6\% of patients fulfilled Hidradenitis Suppurativa Clinical Response criteria with adalimumab at 12 weeks, many responders were not satisfied with their disease control, and secondary loss of Hidradenitis Suppurativa Clinical Response fulfillment occurred in $15.9 \%$ of patients within approximately 3 years. ${ }^{1}$ Without other US Food and Drug Administration-approved HS treatments, some dermatologists have combined adalimumab with methotrexate (MTX) and/or mycophenolate mofetil (MMF) to attempt to increase the duration of satisfactory disease control while on adalimumab. Combining tumor necrosis factor $\alpha$ inhibitors with oral immunosuppressants is a wellestablished approach in psoriasis, psoriatic arthritis, and inflammatory bowel disease; however, to the best of our knowledge, this approach has not been studied for HS., ${ }^{2,3}$

To assess whether there is a role for combining adalimumab with MTX and/or MMF in the treatment of HS, we performed a single-institution, retrospective chart review at the University of Connecticut Department of Dermatology to determine whether patients receiving combination therapy stayed on adalimumab longer than those who received adalimumab monotherapy. All patients receiving adalimumab for the treatment of HS with at least 1 follow-up visit 3 or more months after treatment initiation were included. Duration of freatment with adalimumab was defined as the length of time between initiation and termination of adalimumab, regardless of flares, adverse events, or addition of adjuvant therapy that occurred during this time span. Because standardized rating scales measuring the severity of HS at this time are not recorded routinely at our institution, treatment duration with adalimumab was used as a surrogate for measuring therapeutic success. Additionally, treatment duration is a meaningful end point, as patients with HS may require indefinite treatment. Patients were eligible for inclusion if they were receiving adalimumab for the treatment of HS. Patients were excluded if they were lost to follow-up or had received adalimumab for less than 6 months, as data suggest that biologics do not reach peak effect for up to 6 months in HS. ${ }^{4}$

We identified 116 eligible patients with HS, 32 of whom received combination therapy. Five patients received $40 \mathrm{mg}$ of adalimumab every other week, and 111 patients received $40 \mathrm{mg}$ of adalimumab each week. Patients receiving oral immunosuppressants were more likely to be male and as likely to be biologic naïve compared to patients on monotherapy (Table). The average weekly dose of MTX was $14.63 \mathrm{mg}$, and the average daily dose of MMF was $1000 \mathrm{mg}$. The average number of days between starting adalimumab and starting an oral immunosuppressant was 114.5 (SD, 217; median, 0) days. Reasons for discontinuation of adalimumab included insufficient response, noncompliance, dislike of injections, adverse events, fear

Dr. Zubkov is from the University of Connecticut School of Medicine, Farmington. Drs. Waldman and Grant-Kels are from the Department of Dermatology, University of Connecticut Health Center, Farmington. Dr. Grant-Kels also is from the Department of Dermatology, University of Florida, Gainesville. Dr. Wu is from the Connecticut Convergence Institute for Translation in Regenerative Engineering, University of Connecticut, Farmington. The authors report no conflict of interest.

Correspondence: Reid A. Waldman, MD, UCONN Dermatology Department, 21 South Rd, Farmington, CT 06032 (waldman@uchc.edu). doi: 10.12788 /cutis.0366 
Patient Demographics and Treatment $(\mathrm{N}=116)$

\begin{tabular}{|c|c|c|c|}
\hline Variable & $\begin{array}{l}\text { Adalimumab with concurrent } \\
\text { oral immunosuppressive } \\
\text { medication }\end{array}$ & $\begin{array}{l}\text { Adalimumab without concurrent } \\
\text { oral immunosuppressive } \\
\text { medication }^{\mathrm{a}}\end{array}$ & $P$ value ${ }^{b}$ \\
\hline \multicolumn{4}{|c|}{ Days on adalimumab } \\
\hline Mean & 1012.0 & 503.9 & .0023 \\
\hline SD & 816.7 & 430.0 & \\
\hline \multicolumn{4}{|l|}{ Age, y } \\
\hline Mean & 41.2 & 35.4 & .039 \\
\hline $\mathrm{SD}$ & 13.1 & 13.4 & \\
\hline \multicolumn{4}{|l|}{ Sex, n (\%) } \\
\hline Male & $19(59.4)$ & 25 (30.5) & .0044 \\
\hline Female & $13(40.6)$ & $57(69.5)$ & \\
\hline \multicolumn{4}{|c|}{ Prior biologic use, n (\%) } \\
\hline Yes & $2(6.3)$ & $5(6.4)$ & .99 \\
\hline No & 29 (90.6) & 73 (93.6) & \\
\hline \multicolumn{4}{|c|}{$\begin{array}{l}\text { Other therapy (eg, topicals, antibiotics, } \\
\text { spironolactone), } \mathrm{n}(\%)\end{array}$} \\
\hline Yes & $15(46.9)$ & 39 (51.3) & .67 \\
\hline No & $17(53.1)$ & $37(48.7)$ & \\
\hline
\end{tabular}

of adverse events, other medical issues unrelated to HS, and insurance coverage issues. Patients who ended treatment with adalimumab owing to insurance coverage issues were still included in our study because insurance coverage remains a major determinant of treatment choice in HS and is relevant to the dynamics of medical decision-making.

Statistical analysis was conducted on all patients inclusive of any reason for discontinuation to avoid bias in the calculation of treatment duration. Cox regression analysis was conducted for all independent variables and was noncontributory. Kaplan-Meier methodology was used to assess the duration of treatment of adalimumab with and without concomitant oral immunosuppressants, and quartile survival times were calculated. Quartile survival time is the time point after adalimumab initiation at which $25 \%$ of patients have discontinued adalimumab. We chose quartile survival time instead of average treatment duration to adequately power this study, given our small patient pool.

Although patients receiving adalimumab with oral immunosuppressants had a longer quartile treatment duration (450 days; 95\% CI, 185-1800) than the group without oral immunosuppressants (360 days; 95\% CI, 200-700), neither MTX nor MMF was shown to significantly prolong duration of therapy with adalimumab (log-rank test: $P=.12$ ). Additionally, patients receiving combination therapy were just as likely to discontinue adalimumab as those on monotherapy ( $\chi^{2}$ test: $P=.93$ ). Patients who took both MTX and MMF at different times did show a statistically significant increase in adalimumab quartile treatment duration (1710 days; 95\% CI, 1620 [upper limit not calculable]), but this is likely because these patients were kept on adalimumab while trialing adjunctive medications.

The results of our study indicate that MTX and MMF do not prolong duration of adalimumab therapy, which suggests that adalimumab combination therapy with MTX and MMF may not improve HS more than adalimumab alone, and/or partial responders to adalimumab monotherapy are unlikely to be converted to satisfactory responders with the addition of oral immunosuppressants. Limitations of our study include that it was retrospective, used treatment duration as a surrogate for objective efficacy measures, and relied on a single-institution data source. Additionally, owing to our small sample size, we were unable to account for certain potential confounders, including patient weight and insurance status. Future controlled prospective studies using objective end points are needed to further elucidate whether oral immunosuppressants have a role as an adjunct in the treatment of HS.

\section{REFERENCES}

1. Zouboulis CC, Okun MM, Prens EP, et al. Long-term adalimumab efficacy in patients with moderate-to-severe hidradenitis suppurativa/acne inversa: 3-year results of a phase 3 open-label extension study. J Am Acad Dermatol. 2019;80:60-69.e2. doi:10.1016/j.jaad.2018.05.040

2. Menter A, Strober BE, Kaplan DH, et al. Joint AAD-NPF guidelines of care for the management and treatment of psoriasis with biologics. J Am Acad Dermatol. 2019;80:1029-1072. doi:10.1016/j.jaad.2018.11.057

3. Sultan KS, Berkowitz JC, Khan S. Combination therapy for inflammatory bowel disease. World J Gastrointest Pharmacol Ther. 2017;8:103-113. doi:10.4292/wjgpt.v8.i2.103

4. Prussick L, Rothstein B, Joshipura D, et al. Open-label, investigatorinitiated, single-site exploratory trial evaluating secukinumab, an anti-interleukin-17A monoclonal antibody, for patients with moderateto-severe hidradenitis suppurativa. Br J Dermatol. 2019;181:609-611. 